\title{
Artificial Insemination and Cryopreservation of Boar Semen: Current State and Problematics
}

\author{
Simona Kajabova ${ }^{1}$, Helena Silva ${ }^{1}$, Loide Valadao $^{1}$, Fernando \\ Moreira da Silva ${ }^{1 *}$ \\ ${ }^{1}$ University of Azores - Faculty of Agrarian Sciences and Environment - \\ Angra do Heroismo, Portugal \\ *Corresponding author: Fernando Moreira da Silva: Joaquim.fm.silva@uac.pt
}

\section{OPEN ACCESS}

Citation: Kajabova S., Silva H., Valadao L., Moreira da Silva F. (2020) Artificial Insemination and Cryopreservation of Boar Semen: Current State and Problematics. Open Science Journal 5(2)

Received: $31^{\text {st }}$ January 2020

Accepted: $27^{\text {th }}$ February 2020

Published: $3^{\text {rd }}$ June 2020

Copyright: (C) 2020 This is an open access article under the terms of the Creative Commons Attribution License, which permits unrestricted use, distribution, and reproduction in any medium, provided the original author and source are credited.

Funding: The author(s) received no specific funding for this work

Competing Interests: The author has declared that no competing interests exists.

\begin{abstract}
:
Commercial artificial insemination with boar semen still prefers the usage of refrigerated semen up to 5 days over frozen-thawed, to date. This is because of the uneconomical properties of frozenthawed boar semen, such as low motility, viability, fertility rates and the need for higher semen doses, because of the decreased quality after cryopreservation. Since boar semen is highly susceptive to cold shock damage, the invention of a successful cryopreservation method would be greatly beneficial for the swine industry. This review briefly focuses on the many factors that influence the quality of frozen-thawed boar semen, including the different compositions of extenders, comparison of commercial extenders, freezing and thawing methods (temperature and duration). It could be concluded from the present review that optimum freezing/thawing protocol for swine is not standardized, so far being the current status still considered poor-to-fair.
\end{abstract}

Keywords: Artificial Insemination, Boar semen, Cryopreservation, Extender

\section{Introduction}

Artificial insemination is being used worldwide by swine producers and has a pivotal role on the improvement of global swine production. It has become highly competitive, since the producers are forced to incorporate breeding methods of higher efficiency and profitability, so that productivity increases alongside with cost-reduction [1].Many factors that should be taken into consideration when thinking about the cryopreservation of boar semen includes composition of 
diluents, type and concentration of cryoprotective agent, equilibration time, cooling rate and thawing procedure [2].

Boar semen differs in several aspects from the semen of other domestic animals: the semen is produced in a large volume, it is highly sensitive to cold shock, the viability of the sperm cells is dramatically reduced when exposed to temperatures below $15^{\circ} \mathrm{C}$. Moreover, cryopreservation, because of the very low temperatures, also induces sublethal damage to the spermatozoa, which may result in loss of motility, viability, fertilizing capacity, deterioration of acrosomal and plasma membrane integrity, and damage of deoxyribonucleic acid (DNA) $[2,3]$. Because of that, many researchers have investigated how to improve reproductive performance by artificial insemination (AI) using cryopreserved boar spermatozoa [4]. Actually, AI programmes based on frozen-thawed boar spermatozoa are uneconomical for routine commercial use in the pig industry because the fertility obtained $(<50 \%)$ cannot be compared with the fertility of AI when using fresh or refrigerated semen (nearly $80 \%$ ) or natural service. In addition, variation in freezing susceptibility of spermatozoa between boars is a major factor limiting the use of frozen spermatozoa $[5,6]$.

Although frozen boar semen is available for a long time, a very low proportion of the commercial artificial inseminations are done by using this semen due to its lower survival post thaw resulting in low farrowing rates and litter sizes [7]. While sperm cryopreservation theoretically appears to be the best technology to store boar semen for longer time periods, only $1 \%$ of all AIs conducted worldwide are made using frozen-thawed boar sperm [8]. Utilization of preserved semen for AI in pigs has increased approximately threefold in the past 15 years. More than $99 \%$ of the estimated 19 million inseminations conducted worldwide are made with semen that has been extended in the liquid state and used on the same day or stored at $15-20^{\circ} \mathrm{C}$ up to 7 days post-collection with minimal fertility reduction. Eighty-five percent of all inseminations are conducted on the day of collection or on the following day $[6,9]$.

\section{History}

The first observations concerning low-temperature preservation of spermatozoa date as far back as 1776 , when the Italian physiologist Spallanzani noted that spermatozoa cooled in snow became inactive but revived on warming. Successful cryopreservation protocols date only from the 1950s. In 1949, the single most important development came with the discovery by [10] that glycerol could act as a cryoprotectant for spermatozoa. The initial experiment with glycerol were performed using fowl sperm, but after much effort these were quickly followed by the successful preservation of bull sperm with the first calf conceived by AI using frozen-thawed sperm reported in 1951 [11].Fertility reports with frozen bull spermatozoa led to intensive development of cryopreservation methods that would be applicable for practical insemination purposes in several species (Table 1), including research to develop a method for deep freezing boar semen [12]. 
Table 1. First recorded offspring produced by artificial insemination using frozen-thawed semen in different species [13].

\begin{tabular}{|c|c|}
\hline Species & Date \\
\hline Cows & 1951 \\
\hline Humans & 1953 \\
\hline Pigs & 1957 \\
\hline Horses & 1957 \\
\hline Sheep & 1967 \\
\hline
\end{tabular}

The earliest attempts to perform AI of pigs were made by Ivanoff [14] and later between 1931-1933 by Milovanov, [15] but its true development and wide commercial application in the pig industry did not take place until the 1980s.

\section{Problematics, current state and future importance}

Developing commercially acceptable protocols of boar semen cryopreservation would be in the best interests of the global swine industry. Effective boar semen cryopreservation would enhance herd productivity, assist biosecurity measures, encourage international exchange, facilitate gender selection technology, and allow rational gene banking. Of particular importance to the pig industry, the use of frozen semen would help control transmission of certain pathogens, thereby protecting herd health status [16].

Since depending on their size, individual studs often serve as a supplier to 20 to 200 farms with production of hundreds to thousands of doses of semen each week. The success of the breeding farms relies on the fertility and quality of the semen and the genetic value of the boars at the station [17]. Similar to bull semen, $30-50 \%$ of frozen-thawed boar sperm undergo cell death and a considerable proportion of "live" cells suffer from sub-lethal cryo-damage, which leads to reduced efficiency of sperm transport, shorter survival times in the female tract, and premature membrane destabilization [18].During cryopreservation there are numerous changes that occur and possibly cause irreversible damage to the sperm cell. One of them being the decoating of extracellular matrix components (and lipids) and concomitant coating of proteins (and lipids) from the cryoprotective diluent (milk, albumin or egg yolk). Also, the reduced temperature causes lateral phase separation of lipids and thus a lateral reordering of membrane components and separation of lipid phases, which leads to irreversible changes of membrane proteins, loss or fusion of membrane bilayers and organelle disruption $[19,20,21]$. This all results in weakening of the cell that reduces its ability to withstand future stress [21]. Cryopreservation of boar spermatozoa also appears toalter the sperm nucleus, because it destabilizes the nucleoprotein structure by disrupting disulphide bonds and, to a much less extent, increases DNA fragmentation [22]. Moreover, it has been described that sperm cryopreservation is associated with oxidative damage and reactive oxygen species generation (ROS), which impair normal sperm function and fertilising potential. Boar spermatozoa are highly sensitive to the negative effects of ROS because of the high content of polyunsaturated fatty acids in the plasma membrane and the low antioxidant capacity of seminal plasma [23]. Since spermatozoa discard most of their cytoplasm during the terminal phases of differentiation, which leads to the lack of important cytoplasmic components such 
as antioxidants, this inevitably causes damage induced by ROS and lipid peroxidation (LPO). Also, sperm manipulation, including cooling and freezing, produces imbalances between antioxidant and pro-oxidant activities, and excessive ROS leads to a lipoperoxidative process [3,23]. Conversely, when the balance between ROS production and detoxification by antioxidants is disrupted, an excess of ROS creates oxidative stress. Furthermore, ROS such as H2O2 are known to lower motility and block oxidative metabolism in spermatozoa. Boar spermatozoa are especially sensitive to peroxidative damage, due to the high content of unsaturated fatty acids in the phospholipids of the plasma membrane and the relatively low antioxidant capacity of boar seminal plasma [12].

Another key issue with freezing boar semen is that variations in freezability can be observed even within the same boar (inter-ejaculate difference) [4].

Even though there has been major progress in cryopreservation of boar semen made over the years, the quality of refrigerated semen still is superior with less variability between boars; allowing for less strict AI management and easier handling on the farm. Moreover, fresh liquid semen provides nearly twice as many semen doses per ejaculate (around 40-50 for traditional, 60-80 doses for post-cervical AI than frozen semen [18]. The biological reason for the necessary large number of sperm per AI-dose can be connected with the weakness of frozenthawed (FT) sperm, which is especially vulnerable during their transit through the long pig female reproductive tract. In comparison with refrigerated-stored semen, 4-6 times more FT-sperm are required per oestrus, implying few AI-doses prepared per ejaculate, a non-economic situation [1]. When compared with AI with fresh-refrigerated sperm, the use of cryopreserved sperm contributes to a 10 to $50 \%$ reduction in farrowing rates and, importantly, an averagelitter size of 1.5 to 3.0 fewer piglets [9].However, although frozen semen is used to a very limited extent for genetic improvement, for conservation of rare or valuable genetic material and for overseas import-export, everyday commercial use is nonexistent[13].

\section{From collection to thawing}

The principles of cryopreservation are that when cells are exposed to a solution containing a cryoprotectant (CPA), and the differences in the osmotic pressure of the solution inside and outside the cell will cause an initial diffusion of water from the cell to equilibrate the osmotic pressures. The freezing point of the external solution is dependent on the CPAs present. During the freezing procedures, once the cooling starts the external media freezes before the cells and as water leaves to the exterior causing their dehydration. This leads to an increase concentration of solutes inside, causing a thermodynamic equilibrium avoiding formation of lethal internal ice crystals [24]. Eventually, at a sufficiently low temperature (below about $-80^{\circ} \mathrm{C}$ ), the remaining highly concentrated solutes as well as highly viscous solution within and outside the cells turns into a glassy matrix, which is the fairly stable form for long-term preservation [20]. Cooling and thawing rates are thus the most critical variables influencing sperm survival being this an important requirement for an optimal sperm cryopreservation protocol. Therefore, sperm preservation protocols may differ among animal species owing to their inherent particularities that require changes in extenders used for refrigeration and freezing. Extenders for freezing sperm cells contain buffers, carbohydrates (glucose, lactose, raffinose, saccharose and trehalose), salts 
(sodium citrate, citric acid), egg yolk and antibiotics [25]. As spermatozoa from different species have different reactions to different cooling and thawing, determination of the rates of water permeability using differential scanning calorimetry has confirmed, for instance, that the optimal cooling rate for freezing boar spermatozoa is $-30{ }^{\circ} \mathrm{C} / \mathrm{min}$ when a standard glycerol concentration is used [26], while for cattle bulls optimum cooling rate was between 76 and $140^{\circ} \mathrm{C} / \mathrm{min}$. [27].

\section{Semen collection and evaluation}

The most common method of boar semen collection is the gloved-hand technique, but newer technologies incorporating a collection dummy that allows for hands-free (automatic) semen collection are gaining popularity. With either technique, digital pressure is applied to the boar's glans penis to stimulate erection and ejaculation. Removal of preputial fluid prior to collection and clipping sheath hairs areroutine management practices thatcan greatly assist in reducing the risk of contamination of semen during collection [28]. During collection the semen is filtered through gauze and only sperm rich fractions are collected. Within 30 min after collection semen characteristics (Table 2) such as volume, $\mathrm{pH}$, sperm motility, concentration, percentage of live and dead sperm and morphology are determined [2].

Table 2. Minimum requirements for use of fresh boar semen for artificial insemination [28].

\begin{tabular}{|c|c|}
\hline Semen variable & Descriptor/ value \\
\hline Appearance & Milky to creamy consistency \\
\hline Gross motility (unextended) & $\geq 70 \%$ (if used by 48 hours) \\
& $\geq 80 \%$ (if used after 72 hours) \\
\hline Abnormal morphology & $\leq 25 \%$ (includes cytoplasmic droplets) \\
\hline Cytoplasmic droplets & $\leq 15 \%$ (proximal and distal droplets) \\
\hline
\end{tabular}

After thawing, semen evaluation must be assessed within 30 min to determine the number of sperm required for insemination [29]. Motility assessment can be done either by subjective estimation using a microscope or objectively with computer assisted semen analysis (CASA) system. In boar, it is usual to obtain motility of $80 \%$ and higher. Good quality boar semen is normally too opaque to be evaluated for concentration in the most commonly used methods. For such reason, to determine concentration it must be diluted with an isotonic solution using calibrated pipettes. Also, visual assessment is important when evaluating the quality since normal boar ejaculated should exhibit less than 20-25\% abnormal sperm [28]. Another important factor when judging the quality of frozen boar semen is to evaluate the acrosome status of live cells as only intact acrosome can initiate the binding to the zona pellucida: a premature acrosome reaction makes the spermatozoa not able to fertilize the oocyte [30]. 


\section{Additives and extenders}

Semen extenders provide nutritional and metabolic support for stored sperm. Glucose is the predominant energy source, electrolytes assist in the regulation of osmotic pressure, buffers are involved in neutralization of metabolic wastes and maintenance of $\mathrm{pH}$ and antimicrobials are added to control any bacterial contamination. Several different factors determine the longevity of stored semen, including the individual boar characteristics, particular semen extender, sperm concentration and storage temperature, among others [28]. Up to 1990, extenders were able to maintain fertility for 3 days before significant declines were observed. Extension of fertile life beyond 3 days was a major obstacle until the addition of BSA to the extender, preserving motility and viability up to 5 days [17]. Most extenders for boar semen use standard lactose- egg yolk base for cooling or freezing. In general, the freezing media often contains laurylsulphate, Orvus es Paste- (OEP) as surfactant and glycerol as cryoprotectant (at the final concentration of $2-3 \%$ ) [31].

\section{Additives}

A promising approach for improving cryopreserved boar sperm fertility has been the use of additives included prior to cooling, during processing for freezing and when thawing, to retain normal cell characteristics important to fertility. In a review paper published by [29] it has been postulated that the most common additives are antioxidants, hormones, sugars, proteins and lipids (Table 3).The discovery of the cryoprotective actions of glycerol allowed freezing of spermatozoa and although hundreds of potential cryoprotective agents (CPAs) have subsequently been examined, glycerol has remained, almost without exception, the cryoprotectant of choice for spermatozoa from almost all species [13].

Table 3. Different cryoprotective agents used to freeze cells [32].

\begin{tabular}{|c|c|c|c|c|}
\hline $\begin{array}{l}\text { Alcohols and } \\
\text { Derivates }\end{array}$ & $\begin{array}{l}\text { Sugar and Sugar } \\
\text { alcohols }\end{array}$ & Polymers & $\begin{array}{l}\text { Sulfoxides and } \\
\text { Amides }\end{array}$ & Amines \\
\hline Methanol $^{\mathrm{b}}$ & Glucose $^{\mathrm{b}}$ & Polyethylene glycol (PEG)c & Dimethyl sulfoxide ${ }^{\mathrm{e}}$ & Proline $^{\mathrm{c}}$ \\
\hline Ethanol $^{\mathrm{a}}$ & Galactose $^{\mathrm{b}}$ & $\begin{array}{l}\text { Polyvinyl pyrrolidone } \\
(\mathrm{PVP})^{\mathrm{b}}\end{array}$ & Acetamide $^{\mathrm{b}}$ & Glutamine $^{\mathrm{b}}$ \\
\hline Glycerol $^{\mathrm{d}}$ & Lactose $^{\mathrm{a}}$ & Dextrans $^{c}$ & Formamide $^{\mathrm{b}}$ & Betaine $^{b}$ \\
\hline 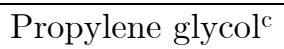 & Sucrose $^{\mathrm{a}, \mathrm{c}}$ & Ficoll $^{\mathrm{c}}$ & Dimethyl acetamide ${ }^{a}$ & \\
\hline \multirow[t]{4}{*}{ Ethylene glycolc } & Trehalose $^{c}$ & Hydroxeythyl starch ${ }^{c}$ & & \\
\hline & Raffinose $^{c}$ & $\begin{array}{l}\text { Serum proteins (complex } \\
\text { mix })^{c}\end{array}$ & & \\
\hline & Mannitola,b & $\begin{array}{l}\text { Milk proteins (complex } \\
\text { mix })^{\mathrm{a}, \mathrm{b}}\end{array}$ & & \\
\hline & Sorbitol $^{\mathrm{a}}$ & Peptones $^{\mathrm{a}}$ & & \\
\hline
\end{tabular}

a Effective in prokaryotic cells

b Effective to a limited degree in eukaryotic cells

c Moderately effective in eukaryotes; often in combination

d Very effective in a defined number of cell types

e Highly effective and widely used acroos all classes of cells 
Extracellular metabolites such as lactate, pyruvate, citrate and even triglycerides have been reported as external energy sources for sperm from species such as boars [33,34,35]. According to [36], non-permeable cryoprotectants, such as sugars, protect the cell membrane from volume changes upon freezing and thawing, whereas permeable cryoprotectants, such as glycerol, are very effective in lowering the intracellular water freezing point and reduce intracellular ice crystal formation. Sugars are not able to diffuse across the plasma membrane, creating an osmotic pressure that induces cell dehydration and a lower incidence of intracellular ice formation. Some authors stated that the cryoprotective effects of the sugars on sperm cells may differ according to their molecular weight: sugars having higher molecular weights exhibit a greater cryoprotective effect $[37,38]$. Nowadays lactose is the sugar most commonly used for boar sperm cryopreservation, due to the good results it provides [39]. According to [36] solutions of lactose, trehalose and powdered coconut water associated with glycerol, produces higher quality of sperm than the other solutions after swine semen cryopreservation using a controlled cooling curve. The addition of sugars to the freezing extender has also beneficial effects on sperm membrane integrity. Furthermore [40], concluded that the addition of trehalose significantly improved the freezability of boar semen, with higher survival and fertility rates. Studies [41] suggest that addition of low-density lipoproteins (LDL) to the extender, yields better results, such ashigher percentage of motility, acrosome and membrane integrity, than when the trehalose is added.

In recent years, the addition of antioxidants such as GSH (glutathione) to ram sperm, [42], green tea extract to boar sperm [43] taurine and cysteine to bovine sperm has been shown to protect sperm against the harmful effects of ROS and to improve post-thaw sperm motility, viability and fertility [3]. The success of the boar sperm cryopreservation was dramatically increased when the detergent sodium dodecyl sulphate (SDS; later known as Equex-STMR) was included in the cryopreservation protocol [44]. According to [3], the addition of antioxidants (carnitine and inositol) in certain doses improve sperm motility but not fertility not did it reduce LPO in post-thawed bovine sperm.

Antibiotic inclusion in the extenders has important effects on sperm survival in refrigerated semen because bacteria are commonly associated with boar ejaculates and can grow quite well in the extenders. To prevent bacterial transmission in semen, antibiotics are often added in combinations to inhibit different strains of bacteria from growing which could also have spermicidal effects [17]. A broad-spectrum antibiotic as a protection against microorganism proliferation is included in most diluents, being penicillin (500-1000 iu/ml) and streptomycin $(0.5-1.0 \mathrm{mg} / \mathrm{ml})$ the most commonly used [45].

\section{Extenders}

The extenders used for semen preservation of domestic species must have suitable $\mathrm{pH}$ and buffering capacity, proper osmolality and should be able to protect sperm cells from cryogenic injury. Commonly, sperm cryopreservation extenders include a non-permeating cryoprotectant (milk or egg yolk), a penetrating cryoprotectant (glycerol, ethylene glycol, or dimethyl sulfoxide), a buffer (Tris or Test), one or more sugars (glucose, lactose, raffinose, saccharose, or trehalose), salts (sodium citrate, citric acid) and antibiotics (penicillin, streptomycin) [25]. For freezing, the routine protocol boar semen consists of a two-step extension method using an egg yolk based extender. First the semen is diluted with an extender without glycerol (Fraction 1) and cooled to $5{ }^{\circ} \mathrm{C}$, then it 
is diluted with an extender containing glycerol, resulting in a final concentration of $3 \%[40]$.

Diluents for fresh semen can be divided into two major groups: those designed for short-term preservation (1-3 days), and for long term semen preservation (over 4 days) (Table 4) [19]. Long-term extenders, whose formulas are mostly proprietary, may contain improved buffering agents, antioxidants, and antibiotics [17].

Table 4: Examples of short- and long-term commercial diluents [19].

\begin{tabular}{|c|c|}
\hline Short-term extenders (1-3 days) & Long-term extenders (over 4 days) \\
\hline Beltsville Liquid (BL-1) & Acromax \\
\hline Beltsville Thawing Solution (BTS) & Androhep \\
\hline Illinois Variable Temperature (IVT) & Modena \\
\hline Kiev & MR-A \\
\hline Vital $\mathbb{R}$ & MULBRRY III \\
\hline & Reading \\
\hline & X-Cell \\
\cline { 2 - 2 } & Zorlesco \\
\cline { 2 - 2 } & ZORPVA \\
\cline { 2 - 2 }
\end{tabular}

Beltsville Thawing Solution is a fairly simple extender widely used for storage of boar semen up to 3 days. It contains a small amount of potassium, a characteristic that allows the preservation of the sodium potassium pump [46]. For boar semen freezing, extenders containing lactose-egg yolk 20\% (v:v) egg yolk, $80 \%$ of $11 \%$ L-lactose solution are very commonly used (e.g. [40],[47]).

In a study presented by [46] no significant differences were observed between extenders (BTS, MR-A, X-Cell) in their capacity to preserve viability, motility or chromatin stability of spermatozoa from normal, fertile boars for $96 \mathrm{~h}$ storage at $17{ }^{\circ} \mathrm{C}$. This result is important, as in the swine industry, semen is most often preserved for shorter periods of time and hardly ever for more than $120 \mathrm{~h}$ (5 days). The team of Dziekońska on 2013 [48] studied different long-term extenders (Androhep, DILU-Cell, Safe Cell Plus ${ }^{\top \mathrm{T}}$ and Vitasem LD), describing that Androhep and Safe Cell Plus extenders, had greater ability to preserve the metabolic activity and membrane integrity of boar spermatozoa during long-term refrigerated semen. These extenders preserved higher proportions of spermatozoa with intact-membrane and normal apical ridge acrosomes, and had higher total motility when stored for 10 days at $17{ }^{\circ} \mathrm{C}$. Similar extenders were used by [49] on frozen-thawed boar semen, and they also observed higher motility when using Safe Cell Plus ${ }^{T M}$, with the difference that Androhep yielded worse results.

\section{Freezing and thawing}

With the common cryopreservation protocols, spermatozoa suspended in cryoprotective medium in French straws $(0.25$ or $0.5 \mathrm{~mL})$ or maxi straws $(5 \mathrm{~mL})$ are cooled in the vapour phase over the surface of liquid nitrogen. Once the latent heat of fusion, released on the initial ice crystallisation, has been dissipated, the cooling rate curve in such protocols is more rapid at the outset reducing as the temperature in the vapour phase is approached [50]. In another 
technique, less used, semen is frozen in pellets. Briefly, after equilibration in a refrigerator at $5^{\circ} \mathrm{C}$ for $2 \mathrm{~h}$, sperm suspensions are frozen in $0.2-\mathrm{ml}$ pellets on a copper network ( $1 \mathrm{~cm}$ above the liquid nitrogen level). The sperm pellets are then held for 30 s on the coppernetwork and transferred into the liquid nitrogen tank for storage [51].

Cooling and freezing

The high susceptibility of boar spermatozoa to cold shock results in a loss of viability of an increasing number of spermatozoa, occurring when freshly ejaculated spermatozoa is quickly cooled from body temperature to temperatures below $15^{\circ} \mathrm{C}[6]$. Chilling injury can be reduced by the inclusion of egg yolk in the medium at temperatures of $15{ }^{\circ} \mathrm{C}$ and lower. It can be also prevented in most freezing protocols by allowing a slow and gradual cooling (approximately 1 ${ }^{\circ} \mathrm{C} / \mathrm{min}$ ) from 15 to $5{ }^{\circ} \mathrm{C}$ [52]. Of considerable importance in the cooling regime is the cooling rate through the critical temperature range, defined as the range when ice crystal formation and consequent cell dehydration is occurring. During this (approximately from -5 to $-50{ }^{\circ} \mathrm{C}$ ), the cooling rate determines whether the cells remain in equilibrium with their extracellular environment or become progressively supercooled with the increasing possibility of intracellular ice formation [50] (Figure 1).

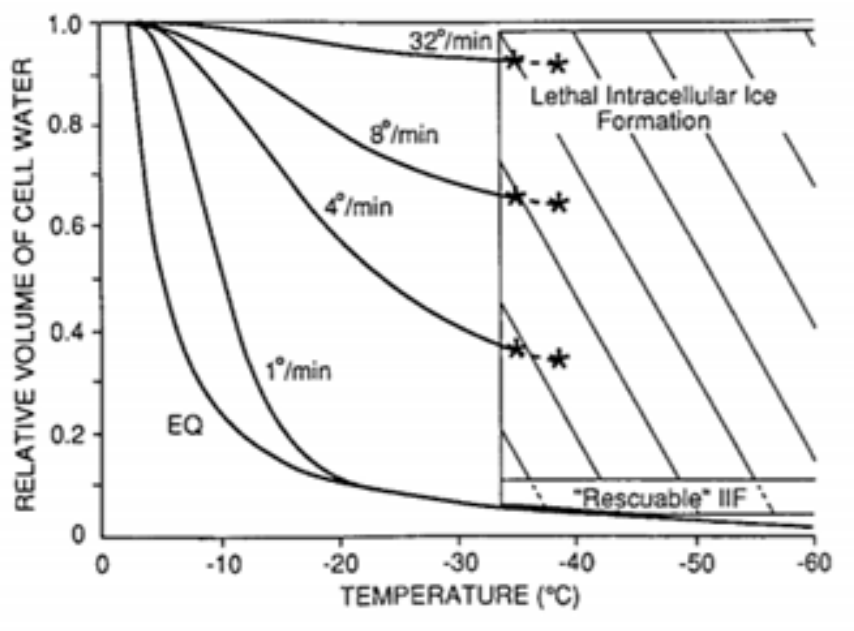

Figure 1: The importance of proper cooling rates: In case the cooling process is too fast, it will result in the intracellular ice formation (IIF) of lethal crystals

[53]. EQ represents a cell cooled infinitely slowly

The potential for such intracellular ice formation increases if the osmotic potential inside the cell becomes disrupted from that in the surrounding medium on a kinetic basis, usually during faster cooling when there is insufficient time for water to move down the chemical potential gradient from the (relatively) more dilute intracellular solution to the concentrated extracellular medium [20].The result of the study published by [7] clearly indicated that the controlled freezing could render higher live sperm, live intact acrosome and intact plasma membrane in comparison with freezing followed in a conventional method. 
Thawing

The factor that could alter the optimal temperature and duration of thawing for cryopreserved boar sperm might could be the packaging material used, form and volume, to optimize the surface area-to-volume ratio to allow for uniform thawing. Cryopreserved boar sperm is usually thawed in the range of $37{ }^{\circ} \mathrm{C}$ to 70 ${ }^{\circ} \mathrm{C}$ for 5 to 40 seconds. The temperature for lower-volume straws $(0.25-0.5 \mathrm{~mL})$ is usually in the range from 10 to 20 seconds at $50{ }^{\circ} \mathrm{C}$ or for 5 to 10 seconds at 70 ${ }^{\circ} \mathrm{C}$, while greater-volume straws $(5-7 \mathrm{~mL})$ are typically thawed for 40 to 50 seconds at the temperature $37{ }^{\circ} \mathrm{C}$ and $50{ }^{\circ} \mathrm{C}$ [29]. Faster thawing with higher temperatures for shorter period of time could be generally considered to be the most effective, although it must not allow any overheating of the sperm [45]. Thawing methods are thus variable and may be performed in water baths when the insemination occurs within 10-30 min after.

\section{Conclusion}

In conclusion, the swine industry would greatly benefit from the discovery of a cryopreservation method that would adequately preserve the quality of frozenthawed boar semen. The ongoing extensive research on the theme of boar semen preservation, and the production of new commercial extenders each year, gives a promising chance that in the next couple of years, the methods of swine semen cryopreservation will greatly increase the post- thaw quality.

\section{Acknowledgments}

This project was financed in $85 \%$ by FEDER and in $15 \%$ with regional funds through the Programa Operacional Açores 2020 (Operational Program Azores 2020), in scope of the project "CryotecACORES-01-0145-FEDER-000092" .

\section{References:}

1.Roca J, Parrilla I, Martinez HR, Gil MA, Cuello C, Vazquez JM, et al. Approaches Towards Efficient Use of Boar Semen in the Pig Industry. Reproduction in Domestic Animals. 2011;46: 79-83.

2.Katlov II 2012a. Current Frontiers in Cryopreservation InTech 462 pp.

3.Bucak MN, Tuncer PB, Sarızkan S, Baspinar N, Taspinar M, Çoyan K, et al. Effects of antioxidants on post-thawed bovine sperm and oxidative stress parameters: Antioxidants protect DNA integrity against cryodamage. Cryobiology. 2010;61: 248-253.

4.Okazaki T, Shimada M. New strategies of boar sperm cryopreservation: Development of novel freezing and thawing methods with a focus on the roles of seminal plasma. Animal Science Journal. 2012;83: 623-629.

5.Medrano A, Holt WV, Watson PF. Controlled freezing studies on boar sperm cryopreservation. Andrologia. 2009;41: 246-250.

6.Johnson LA, Weitze KF, Fiser P, Maxwell WM. Storage of boar semen. Animal Reproduction Science. 2000;62: 143-172.

7.Baishya SK, Biswas RK, Kadirvel G, Deka BC, Kumar S, Sinha S, et al. Effect of conventional and controlled freezing method on the post thaw characteristics of boar spermatozoa. Animal Reproduction Science. 2014;149: 231-237.

8. Yeste M. Recent Advances in Boar Sperm Cryopreservation: State of the Art and Current Perspectives. Reproduction in Domestic Animals. 2015;50: 71-79. 
9.Benson JD, Woods EJ, Walters EM, Critser JK. The cryobiology of spermatozoa. Theriogenology. 2012;78: 1682-1699.

10.Polge C, Smith AU, Parkes AS. Revival of spermatozoa after vitrification and dehydration at low temperatures. Nature. 1949; 164:666.

11.Stewart DL. Storage of Bull Spermatozoa at Low Temperatures Vet. Record. 1951;63: 65

12.Großfeld R, Sieg B, Struckmann C, Frenzel A, Maxwell WM, Rath D. New aspects of boar semen freezing strategies. Theriogenology. 2008;70: 1225-1233.

13.Curry MR. Cryopreservation of semen from domestic livestock. Reviews of Reproduction. 2000;5: 46-52.

14.Ivanoff, E. I. On the use of artificial insemination for zootechnical purposes in Russia. The Journal of Agricultural Science. 1922;12: 244-256.

15.Milovanov VK. Artificial Insemination of Livestock in the U.S.S.R. Trans. By Birron A and Cole ZS. Washington, DC: S Monson, Jerusalem Tech. Services, US Dept Commerce; 1964. 152.

16.Bailey JL, Lessard CH, Jacques J, Breque CH, Dobrinski I, Zeng W, et al. Cryopreservation of boar semen and its future importance to the industry Theriogenology. 2008;70: 1251-1259

17.Knox RV. Artificial insemination in pigs today. Theriogenology. 2016;85: 83-93.

18. Waberski D, Riesenbeck A, Schulze M, Weitze KF, Johnson L. Application of preserved boar semen for artificial insemination: Past, present and future challenges. Theriogenology. 2019;137: $2-7$.

19.Gadea J. Review: Semen extenders used in the artificial insemination of swine. Spanish Journal of Agricultural Research. 2003;1: 17-27.

20.Fuller BJ. Cryoprotectants: the essential antifreezes to protect life in the frozen state. CryoLetters. 2004;25: 375-388.

21.Leahy T, Gadella BM. Capacitation and Capacitation-like Sperm Surface Changes Induced by Handling Boar Semen. Reproduction in Domestic Animals. 2011;46: 7-13.

22.Yeste M, Estrada E, Casas I, Bonet S, Rodríguez-Gil JE. Good and bad freezability boar ejaculates differ in the integrity of nucleoprotein structure after freeze-thawing but not in ROS levels. Theriogenology. 2013;79: 929-939.

23.Luño V, Gil L, Olaciregui M, González N, Jerez RA, Blas I. Rosmarinic acid improves function and in vitro fertilising ability of boar sperm after cryopreservation. Cryobiology. 2014;69: 157-162.

24.Skinner MK. Encyclopedia of Reproduction. 2nd Edition. Waltham: Elsevier Inc. 2018. p. 3868.

25.Barbas JP, Mascarenhas RD. Cryopreservation of domestic animal sperm cells. Cell Tissue Bank. 2009;10: 49-62.

26.Devireddy RV, Fahrig B, Godke RA, Leibo SP. Subzero Water Transport Characteristics of Boar Spermatozoa Confirm Observed Optimal Cooling Rates. Molecular Reproduction and Development. 2004;67: 446-457.

27.Woelders H. Fundamentals and recent development in cryopreservation of bull and boar semen. Veterinary Quarterly. 1997;19(3):135-8.

28.Zimmerman JJ, Karriker LA, Ramirez A, Schwartz KJ, Stevenson GW. Diseases of Swine. 10th Edition. John Wiley \& Sons Inc. 2012. p. 1008.

29.Knox RV. The Fertility of Frozen Boar Sperm When used for Artificial Insemination. Reproduction in Domestic Animals. 2015;50: 90-97.

30.Spinaci M, Vallorani C, Bucci D, Bernardini C, Tamanini C, Seren E, et al. Effect of liquid storage on sorted boar spermatozoa. Theriogenology. 2010;74: 741-748.

31.Katlov II 2012b. Current Frontiers in Cryobiology InTech 574 pp ISBN: 978-953-51-0191-8

32.Elliott GD, Wang S, Fuller BJ. Cryoprotectants: A review of the actions and applications of cryoprotective solutes that modulate cell recovery from ultra-low temperatures. Cryobiology. 2017;76: 74-91.

33.Jones AR, Chantrill LA, Cokinais A. Metabolism of glycerol by mature boar spermatozoa. Journal of Reproduction and Fertility. 1992;94: 129-34.

34.Jones AR. Metabolism of lactate by mature boar spermatozoa. Reproduction, Fertility and Development 2000; 12: 355-9.

35.Medrano A, Fernández-Novell JM, Ramió L, Álvarez J, Goldberg E, Rivera MM, et al. Utilization of citrate and lactate through a lactate deydrogenase and ATP-regulated pathway in boar spermatozoa. Molecular Reproduction and Development. 2006;73: 369-78.

36.Silva CG, Cunha ER, Blume GR, Malaquias JV, Báo SN, Martins CF. Cryopreservation of boar sperm comparing different cryoprotectants associated in media based on powdered coconut water, lactose and trehalose. Cryobiology. 2015;70: 90-94.

37.Anchordoguy TJ, Rudolph AS, Carpenter JF, Crowe JH. Modes of interaction of crioprotectants with membrane phospholipids during freezing. Cryobiology. 1987;34: 324-331.

38.Molinia FC, Evans G, Maxwell WM. In vitro evaluation of zwitterion buffers in diluents for freezing ram spermatozoa. Reproduction, Nutrition and Development. 1994;34: 491-500.

39.Gómez-Fernández J, Izquierdoa EG, Tomás C, Mocé E, Mercado E. Effect of different monosaccharides and disaccharides on boar sperm quality after cryopreservation. Animal Reproduction Science. 2012;133: 109-116. 
40.Malo Ca, Gil L, Gonzalez N, Cano R, Blas I, Espinosa E. Comparing sugar type supplementation for cryopreservation of boar semen in egg yolk based extender. Cryobiology. 2010;61: 17-21.

41.Hu JH, Li QW, Jiang ZL, Li WY. Effects of different extenders on DNA integrity of boar spermatozoa following freezing-thawing. Cryobiology. 2008;57: 257-262.

42.Bucak MN, Tekin N. Protective effect of taurine, glutathione and trehalose on theliquid storage of ram semen. Small Ruminant Research. 2007;73: 103-108.

43.Park SH, Yu IJ. Effect of Antioxidant Supplementation in Freezing Extender on Porcine Sperm Viability, Motility and Reactive Oxygen Species. Journal of Animal Reproduction and Biotechnology. 2017;32: 9-15.

44.Buranaamnuay K, Tummaruk P, Singlor J, Rodriguez-Martinez H, Techakumphu M. Effects of Straw Volume and Equex-STM on Boar Sperm Quality after Cryopreservation. Reproduction in Domestic Animals. 2009;44: 69-73.

45.Curry MR. Cryopreservation of Mammalian Semen. In: Day JD, Stacey G. Cryopreservation and Freeze-Drying Protocols. 2nd edition. Totowa: Humana Press Inc; 2007. p. 303-311.

46.Ambrogi M, Ballester J, Saravia F, Caballero I, Johannisson A, Wallgren M, et al. Effect of storage in short- and long-term commercial semen extenders on the motility, plasma membrane and chromatin integrity of boar spermatozoa. International Journal of Andrology. 2006;29: 543-552.

47.Malo Cb, Gil L, Gonzalez N, Martínez F, Cano R, Blas I, et al. Anti-oxidant supplementation improves boar sperm characteristics and fertility after cryopreservation: Comparison between cysteine and rosemary (Rosmarinus officinalis). Cryobiology. 2010;61: 142-147.

48.Dziekońska A, Fraser L, Majewska A, Lecewicz M, Zasiadczyk E, Kordan W. Effect of commercial long-term extenders on metabolic activity and membrane integrity of boar spermatozoa stored at $17^{\circ} \mathrm{C}$. Polish Journal of Veterinary Sciences. 2013;16:517-525.

49.Frydrychová S, Lustyková A, Václavková E, Lipenský J, Rozkot M. Effect of different extenders on quality of frozen-thawed boar semen. Indian Journal Of Animal Research. 2015;49: 851-854.

50.Kumar S, Millar JD, Watson PF. The effect of cooling rate on the survival of cryopreserved bull, ram, and boar spermatozoa: a comparison of two controlled-rate cooling machines. Cryobiology. 2003;46: $246-253$.

51.Dai JJ, Wu CF, Zhang DF, Yin FZ, Zhang TY, Liu D, et al. Some Factors Affecting Freezing of Boar Semen in 5 ml Maxi-straws. Asian-Australian Journal of Animal Science. 2009; 22: 507 515 .

52.Woelders H, Matthijs A, Zuidberg CA, Chaveiro AE. Cryopreservation of boar semen: equilibrium freezing in the cryomicroscope. Theriogenology. 2005;63: 383-395.

53.Mazur P, Seki S, Pinn IL, Kleinhans FW, Edashige K. Extra- and intracellular ice formation in mouse oocytes. Cryobiology. 2005;51: 29-53. 